\title{
RISING TREND OF ESBL PRODUCTION IN UROPATHOGENIC E. COLI CAUSING WORRY? - A STUDY IN A TERTIARY CARE HOSPITAL
}

\author{
Vijaya $^{1}$, Sweetha Nayak Nagar ${ }^{2}$
}

${ }_{1}^{1}$ Assistant Professor, Department of Microbiology, Srinivas Institute of Medical Sciences and Research Centre, Mukka, Mangalore, Karnataka, India.

${ }^{2}$ Assistant Professor, Department of Microbiology, Srinivas Institute of Medical Sciences and Research Centre, Mukka, Mangalore, Karnataka, India.

\section{BACKGROUND}

ABSTRACT

Urinary Tract Infection (UTI) caused by Escherichia coli (E. coli) has become a significant global public health problem. The resistance to $\beta$-lactam antibiotics in $E$. coli further adds to the problem. The pattern of drug resistance among E. coli undergoes considerable variation and consequently the treatment of Urinary Tract Infection (UTI) requires constant updating of the antibiotic sensitivity profile.

The study was thus undertaken to know resistance pattern of E. coli isolates with special reference to production of extended spectrum $\beta$-lactamases (ESBLs) in urinary samples.

\section{MATERIALS AND METHODS}

This is a three-year (2015 - 2017) retrospective descriptive study undertaken in Srinivas Institute of Medical Sciences and Research Centre, Mukka, Mangalore. Standard culture techniques for urine samples were followed. Antibiotic sensitivity test was done by Kirby-Bauer disc diffusion method and interpretation was done following Clinical and Laboratory Standards Institute (CLSI) guidelines. ESBL detection was done by NCCLS phenotypic confirmatory combination disc diffusion method using ceftazidime $(30 \mu \mathrm{g})$ alone and ceftazidime + clavulanic acid $(30 \mu \mathrm{g} / 10 \mu \mathrm{g})$.

\section{RESULTS}

E.coli is the most frequent isolate found in UTI (65.4\% of total isolates) throughout three years. The overall resistance pattern of E.coli exhibited highest resistance against ampicillin (95\%) and amoxyclav (83.3\%) with rising trend in resistance to Cephalosporins and Quinolones in three successive years. The rising trend in ESBL production rate was observed from $27.3 \%$ (2015) to 39.4\% (2017) with least resistance against meropenem (9.9\%), piperacillin/ tazobactam (10\%), nitrofurantoin (11.4\%) and amikacin (12.1\%). It was also observed that $>50 \%$ of ESBL producing E. coli isolates exhibited resistance against cotrimoxazole and Quinolones.

\section{CONCLUSION}

The incidence of the ESBL producing E. coli has been steadily increasing over the past few years. The knowledge on the resistance pattern in a geographical area will help in guiding the appropriate and the judicious use of antibiotics.

\section{KEY WORDS}

Uropathogens, ESBL, E.coli.

HOW TO CITE THIS ARTICLE: Vijaya, Nagar SN. Rising trend of ESBL production in uropathogenic E. coli causing worry?- a study in a tertiary care hospital. J. Evolution Med. Dent. Sci. 2018;7(46):4938-4942, DOI: 10.14260/jemds/2018/1099

\section{BACKGROUND}

Urinary Tract Infection (UTI) is a spectrum of disease caused by microbial invasion of the genitourinary tract that extends from the renal cortex of the kidney to the urethral meatus. ${ }^{1}$ Approximately, $40 \%$ of women and $12 \%$ of men experience at least one symptomatic infection during their lifetime. ${ }^{2}$

It also contributes as the most common nosocomial infection in many hospitals and accounts for approximately $35 \%$ of all hospital-acquired infections. This burden causes serious impact on the socioeconomic life of individuals and

'Financial or Other Competing Interest': None.

Submission 04-10-2018, Peer Review 30-10-2018,

Acceptance 05-11-2018, Published 12-11-2018.

Corresponding Author:

Dr. Sweetha Nayak Nagar,

Assistant Professor,

Department of Microbiology,

Srinivas Institute of Medical Sciences and Research Centre, (SIMS and

RC), Mukka, Mangalore, Karnataka, India.

E-mail: sweetsnayak@yahoo.com

DOI: $10.14260 /$ jemds $/ 2018 / 1099$ also leads to a large proportion of antibacterial drug consumption. ${ }^{3}$ UTIs having E. coli as aetiological agent are common infections with an estimated annual global incidence of at least 250 million cases. ${ }^{4}$

Studies indicate that uropathogens are becoming increasingly resistant to the antibiotics used for the treatment of UTI. As an example, SENTRY- an Antimicrobial Surveillance Program that monitored UTI worldwide over a 4-year period between 1997 and 2000 showed an increase in resistance among UTI. The term uncomplicated UTI refers to the invasion of a structurally and functionally normal urinary tract by a non-resident infectious organism. ${ }^{5} \mathrm{~A}$ complicated UTI is an infection associated with a condition such as a structural or functional abnormality of the genitourinary tract or the presence of an underlying disease that interferes with host defence mechanisms, which increases the risk of acquiring infection or of therapeutic failure. This could include the presence of an indwelling catheter or urinary stent, the presence of an obstructive uropathy of any aetiology or urinary tract modifications such as an ileal loop or pouch. ${ }^{6}$ Most cases of UTI are caused by Gram-negative 
bacilli with $E$. coli accounting for over $90 \%$ of uncomplicated UTIs. Uncomplicated infections can be treated with short courses of antibiotics, while complicated UTIs require longer and more intensive courses of antibiotics. However, resistance to the commonly used antibiotics is increasing and making treatment more difficult. ${ }^{7}$ An important mechanism of antibiotic resistance among $E$. coli is through ESBL production. In particular, ESBL producing E. coli are emerging worldwide. The ESBL producing strains are particularly feared as they are resistant to all Penicillins, Cephalosporins (Including third and fourth generation agents) and to aztreonam.

Major risk factors for colonisation or infection with ESBLproducing organisms are long-term antibiotic exposure, prolonged hospital stays, residence in an institution with high rates of third-generation cephalosporins use, and in whom invasive devices (Urinary catheters, endotracheal tubes and central venous lines) are present for a prolonged duration. ${ }^{8}$ The frequency of detection of ESBL producing strains (Including $E$. coli) is quite variable, but is consistently increasing. ${ }^{9} E$. coli is one of the main ESBL-producing pathogen. ESBL production is transmitted from one bacterium to another through plasmids. These plasmids can carry multiple drug-resistant genes against aminoglycosides, quinolones and sulfamethoxazole at the same time. The phenomenon of multidrug resistance has brought tremendous difficulties to the clinical treatment of infection due to limited therapeutic options.

\section{MATERIALS AND METHODS}

This is a three-year (2015 - 2017) retrospective descriptive study undertaken in Srinivas Institute of Medical Sciences and Research Centre, Mukka, Mangalore. All the Culture and Sensitivity (C/S) reports of urine samples, maintained in the record registers of Microbiology laboratory during the study period were included and analysed. A total of 22,055 urine specimens obtained from outpatients and inpatients were analysed. Urine culture was done by standard loop method, a semi-quantitative method. The organisms isolated from urine culture were identified by conventional biochemical tests. Only the E. coli isolates were considered for this study. Antimicrobial susceptibility test was done by Kirby-Bauer disc diffusion method on Mueller-Hinton agar and the interpretations were carried out according to the Clinical and Laboratory Standards Institute guidelines. Antibiotics against which sensitivity was tested included ampicillin $(10 \mu \mathrm{g})$, amoxyclav $(20 \mu \mathrm{g} / 10 \mu \mathrm{g})$, ceftazidime (30 $\mu \mathrm{g})$, ceftriaxone $(30 \mu \mathrm{g})$, aztreonam $(30 \mu \mathrm{g})$, gentamicin $(10 \mu \mathrm{g})$, amikacin (30 $\mu \mathrm{g})$, ciprofloxacin $(5 \mu \mathrm{g})$, co-trimoxazole $(25 \mu \mathrm{g})$, norfloxacin $(10 \mu \mathrm{g})$, nitrofurantoin $(300 \mu \mathrm{g})$, piperacillin-tazobactam $(100 \mu \mathrm{g} / 10 \mu \mathrm{g})$ and meropenem $(10 \mu \mathrm{g})$. Quality control of media and discs were performed using ATCC E. coli control strain 25922. ESBL production in E. coli was detected routinely by NCCLS phenotypic confirmatory combination disc diffusion method using ceftazidime $(30 \mu \mathrm{g})$ and ceftazidime/ clavulanic acid $(30 \mu \mathrm{g} / 10 \mu \mathrm{g})$. An increase in the inhibition zone diameter of $\geq 5 \mathrm{~mm}$ for a combination disc versus ceftazidime disc alone confirmed ESBL production. Whole of the data were collected and compiled in the year wise manner and compared year wise to see the trend in the resistance pattern.

\section{RESULTS}

Over a period of three years (2015 - 2017), a total of 7127 $(32.3 \%)$ urine samples showed significant growth, in which 4 $658(65.4 \%)$ isolates were E. coli as expected E. coli was the most frequent isolate. The next most frequently isolated bacteria were Klebsiella spp. (15.8\%). However, isolates other than $E$. coli were not considered in our study. Out of 4,658 E.coli isolates 2,705 (58\%) of the isolates were obtained from female patients.

The year wise distribution of urine samples, number of cultures with significant growth and number of isolated E.coli are given in Table 1.

\begin{tabular}{|c|c|c|c|}
\hline & $\begin{array}{c}\text { No. of Urine } \\
\text { Samples }\end{array}$ & $\begin{array}{c}\text { No. of Cultures with } \\
\text { Significant Growth }\end{array}$ & $\begin{array}{c}\text { No. of } \\
\text { E. coli }\end{array}$ \\
\hline 2015 & 4,850 & $1,697(35 \%)$ & $1,052(62 \%)$ \\
\hline 2016 & 7,125 & $2,280(32 \%)$ & $1,527(67 \%)$ \\
\hline 2017 & 10,080 & $3,150(31 \%)$ & $2,079(66 \%)$ \\
\hline $\begin{array}{c}\text { Total } \\
\text { No. }\end{array}$ & $\mathbf{2 2 , 0 5 5}$ & $\mathbf{7 , 1 2 7}(\mathbf{3 2 . 3} \%)$ & $\begin{array}{c}\mathbf{4 , 6 5 8} \\
\mathbf{6 6 5 . 4 \% )}\end{array}$ \\
\hline \multicolumn{4}{|c|}{ Table 1 } \\
\hline
\end{tabular}

All the E. coli $(4,658)$ isolates were subjected to antimicrobial susceptibility testing and ESBL detection. The overall resistance pattern of $E$. coli was highest against ampicillin (95\%) and amoxyclav (83.3\%). There was a rising trend in resistance to cephalosporins $(58.6 \%-70.6 \%$ in ceftriaxone, $60 \%-68 \%$ in ceftazidime) and ciprofloxacin $(57.6 \%-65.5 \%)$ in three successive years. More than $50 \%$ of isolates exhibited resistance against co-trimoxazole throughout three years. However, the resistance to nitrofurantoin, amikacin, piperacillin-tazobactam and meropenem was least (Table 2 and Graph 1).

\begin{tabular}{|c|c|c|c|}
\hline \multirow{2}{*}{ Antibiotic } & $\begin{array}{c}\mathbf{2 0 1 5} \\
\text { E. coli No: } \\
\mathbf{1 0 5 2} \\
\mathbf{R ~ ( \% ) ~}\end{array}$ & $\begin{array}{c}\mathbf{2 0 1 6} \\
\text { E. coli No: } \\
\mathbf{1 5 2 7} \\
\mathbf{R ~ ( \% )}\end{array}$ & $\begin{array}{c}\mathbf{2 0 1 7} \\
\text { E. coli No: } \\
\mathbf{2 0 7 9} \\
\mathbf{R}(\%)\end{array}$ \\
\hline Ampicillin & $976(92.8 \%)$ & $1442(94 \%)$ & $2001(96.2 \%)$ \\
\hline Amoxyclav & $849(80.7 \%)$ & $1288(84.3 \%)$ & $1742(83.8 \%)$ \\
\hline Ceftriaxone & $616(58.6 \%)$ & $1005(65.8 \%)$ & $1468(70.6 \%)$ \\
\hline Ceftazidime & $632(60 \%)$ & $985(64.5 \%)$ & $1414(68 \%)$ \\
\hline Ciprofloxacin & $606(57.6 \%)$ & $908(59.5 \%)$ & $1362(65.5 \%)$ \\
\hline Aztreonam & $515(49 \%)$ & $888(58 \%)$ & $1170(56.3 \%)$ \\
\hline Co-trimoxazole & $553(52.6 \%)$ & $790(51.7 \%)$ & $1096(52.7 \%)$ \\
\hline Norfloxacin & $450(42.8 \%)$ & $683(44.7 \%)$ & $1000(48.1 \%)$ \\
\hline Gentamicin & $419(39.8 \%)$ & $736(48.2 \%)$ & $692(33.3 \%)$ \\
\hline Nitrofurantoin & $130(12.4 \%)$ & $169(11 \%)$ & $211(10.1 \%)$ \\
\hline Amikacin & $121(11.5 \%)$ & $161(10.5 \%)$ & $179(8.6 \%)$ \\
\hline $\begin{array}{c}\text { Piperacillin- } \\
\text { Tazobactam }\end{array}$ & $109(10.4 \%)$ & $132(8.6 \%)$ & $185(8.9 \%)$ \\
\hline Meropenem & $75(7.1 \%)$ & $138(9 \%)$ & $168(8 \%)$ \\
\hline
\end{tabular}

Table 2. (Year Wise Resistance Pattern of E. coli Isolates) 


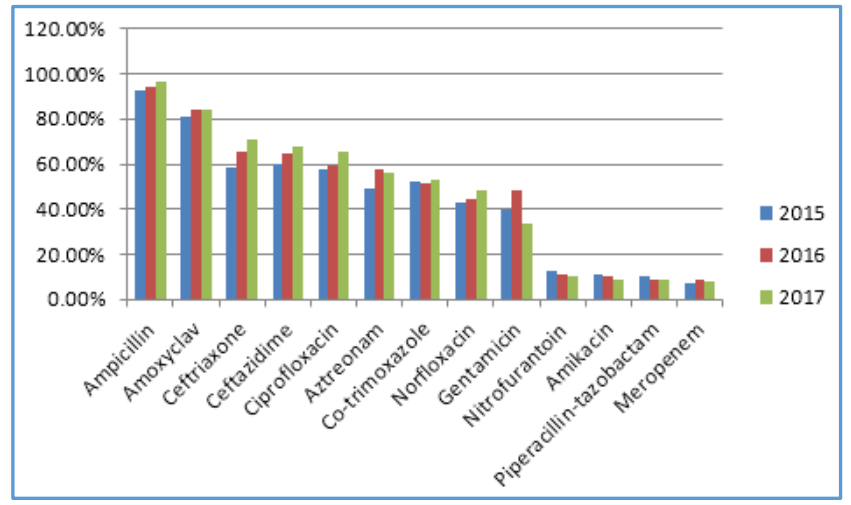

Graph 1. (Year Wise Resistance Pattern of E. coli Isolates)

The results of ESBL producing E. coli were analysed year wise, which showed that 287 (27.3\%) were ESBL producers out of 1,052 isolated E. coli in the year 2015, 537 (35.2\%) out of 1527 E. coli in the year 2016 and 819 (39.4\%) out of 2,079 E. coli in 2017 showing rising trend in ESBL rate which increased significantly from $27.3 \%$ to $39.4 \%$ between 2015 2017 (Table 3 and Graph 2).

\begin{tabular}{|c|c|c|c|}
\hline Year & E. coli No. & ESBL producing E. coli & ESBL (\%) \\
\hline 2015 & 1,052 & 287 & $27.3 \%$ \\
\hline 2016 & 1,527 & 537 & $35.2 \%$ \\
\hline 2017 & 2,079 & 819 & $39.4 \%$ \\
\hline \multicolumn{3}{|c|}{ Table 3. Occurrence of ESBL in E. coli in the Years } \\
2015 - 2017 \\
\hline
\end{tabular}

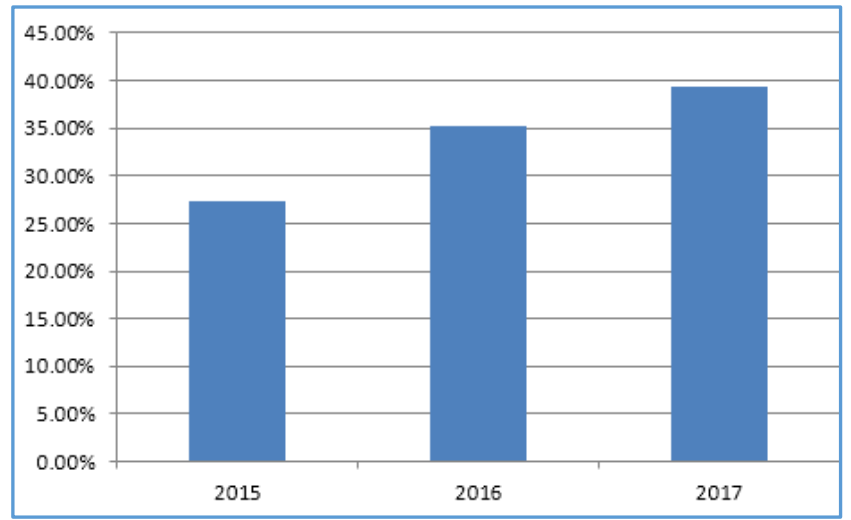

Graph 2. Occurrence of ESBL in

E. coli in the Years 2015 - 2017

Year wise analysis of resistance pattern in ESBL producing E. coli revealed lowest percentage of resistance against meropenem $(10 \%, 10.6 \%, 9.4 \%)$, piperacillintazobactam $(11.2 \%, 10 \%, 9.6 \%)$, amikacin $(15.6 \%, 11.5 \%$, $12.5 \%)$ and nitrofurantoin (13\%, 12.2\%, 10.4\%). Surprisingly, the resistance to nitrofurantoin was found to be decreased over three consecutive years. It was observed that $>50 \%$ ESBL producing E. coli isolates were resistant to cotrimoxazole $(59.8 \%, 63.2 \%, 61.2 \%)$ and fluoroquinolones. There was rising trend in resistance against fluoroquinolones (Ciprofloxacin 67.2\%, 73.5\%, 74.8\% and norfloxacin 50.2\%, $51.2 \%, 55.6 \%$ ) in three successive years.

\begin{tabular}{|c|c|c|c|}
\hline \multirow{3}{*}{ Antibiotic } & $\mathbf{2 0 1 5}$ & $\mathbf{2 0 1 6}$ & $\mathbf{2 0 1 7}$ \\
\cline { 2 - 4 } & $\begin{array}{c}\text { ESBL E. coli } \\
\text { No: 287 } \\
\text { R (\%) }\end{array}$ & $\begin{array}{c}\text { ESBL E. coli } \\
\text { No: 537 } \\
\text { R (\%) }\end{array}$ & $\begin{array}{c}\text { ESBL E. coli } \\
\text { No: 819 } \\
\text { R (\%) }\end{array}$ \\
\hline Ciprofloxacin & $193(67.2 \%)$ & $395(73.5 \%)$ & $613(74.8 \%)$ \\
\hline Co-trimoxazole & $172(59.8 \%)$ & $339(63.2 \%)$ & $501(61.2 \%)$ \\
\hline Norfloxacin & $144(50.2 \%)$ & $275(51.2 \%)$ & $455(55.6 \%)$ \\
\hline Gentamicin & $128(44.5 \%)$ & $228(42.5 \%)$ & $321(39.2 \%)$ \\
\hline Nitrofurantoin & $37(13 \%)$ & $66(12.2 \%)$ & $85(10.4 \%)$ \\
\hline Amikacin & $34(15.6 \%)$ & $62(11.5 \%)$ & $103(12.5 \%)$ \\
\hline $\begin{array}{c}\text { Piperacillin- } \\
\text { Tazobactam }\end{array}$ & $32(11.2 \%)$ & $54(10 \%)$ & $79(9.6 \%)$ \\
\hline Meropenem & $29(10 \%)$ & $57(10.6 \%)$ & $77(9.4 \%)$ \\
\hline Table 4. Year Wise Resistance Pattern of ESBL producing \\
E.coli \\
\hline \multicolumn{4}{|c|}{} \\
\hline
\end{tabular}

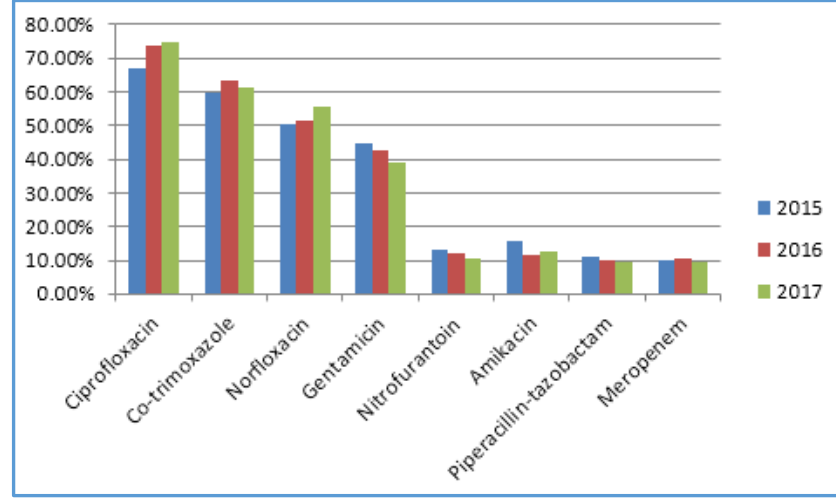

Graph 3. Year Wise Resistance Pattern of ESBL producing E. coli

\section{DISCUSSION}

Infection of the urinary tract is one of the most common infectious diseases and it would affect all age groups including men, women and children worldwide.10 A total of 22,055 non-repetitive urine specimens were analysed during the study period (2015 - 2017). Of these 7,127 (32.3\%) urine samples showed significant growth. Similar growth rates were reported $(30.8 \%)$ in a study by Baral P et al.11 The growth rate was higher (41.7\%) when compared to our study in a study done by Bhatt $\mathrm{C}$ et al. ${ }^{12}$ Data analysis revealed a much higher percentage of women (58\%) to be suffering from UTI as compared to men (42\%). The men are usually less prone to UTI as compared to females owing to the longer course of the urethra and bacteriostatic properties of prostate secretions.

The antimicrobial susceptibility patterns have changed overtime, but the spectrum of agents causing UTI has remained relatively constant with $E$. coli being the most common isolate. ${ }^{13}$ Earlier studies from different regions of India and from other countries have reported that the most prevalent UTI pathogen was E. coli followed by Klebsiella spp. ${ }^{14}$ Similarly, in our study E. coli $(65.4 \%)$ is the most common pathogen isolated in both inpatients and outpatients of both sexes followed by Klebsiella spp. (15.8\%), which goes in accordance with the study done by Chaudhari et al. ${ }^{15}$

Among all 4,658 E. coli isolates highest resistance was noticed against ampicillin (95\%) and amoxyclav (83\%). There was a rising trend in resistance to cephalosporins from $58.6 \%$ (2015) to $70.6 \%$ (2017) for ceftriaxone, 60\% (2015) to $68 \%$ (2017) for ceftazidime in three successive years, which could be due to frequent usage of these antibiotics for 
other infections in the institution. A trend of increasing resistance to ciprofloxacin $(57.6 \%$ - 65.5\% from 2015 - 2017) was found in our study. Fluoroquinolones have a wide variety of indications, permeate most body compartments and are ubiquitously prescribed, accounting for the emergence of their resistance. 16 Similar increasing trend in resistance to cephalosporins and quinolones are found in the study done by Jeyasekharan DD et al. ${ }^{17}$ On an average resistance to cotrimoxazole in our study was $52.4 \%$, which could be attributed to their wide usage for a variety of other indications. Resistance to gentamicin (39.7\%) was higher than amikacin (9.9\%) in our study, which goes in accordance with the study done by Kulkarni SR et al ${ }^{18}$ with $90.89 \%$ sensitivity to Amikacin (9.11\% resistance) and 59.24\% sensitivity to Gentamicin ( $40.76 \%$ resistance). This is in contrast to study done by Revathy Saravanan et al,19 in which isolates were more sensitive to gentamicin compared to amikacin. This supports the possibility of changing sensitivity pattern with time difference. The decreasing trend in resistance to amikacin $(11.5 \%-8.6 \%)$ was noticed in our study. This could be due to being injectables the use of aminoglycosides are restricted in the community care setting and hence might have shown better sensitivity rates. The overall resistance to nitrofurantoin was least (11\%) throughout the three years. Re-emergence of $E$. coli sensitivity to nitrofurantoin is probably due to non-usage of the drug for a long period of time. Our findings are similar to other Indian studies, which have also demonstrated nitrofurantoin as an appropriate agent for first-line treatment of community acquired UTIs. Given the fact that nitrofurantoin has no role in the treatment of other infections, it can be administered orally and is highly concentrated in urine. It may therefore be the most appropriate agent for empirical use in uncomplicated UTI.

Our study exhibited the rising trend in ESBL production rate from $27.3 \%$ (2015) to $39.4 \%$ (2017). Such rising trend in ESBL rate is of considerable significance, because ESBLs have the ability to render all $\beta$-lactam rings containing antibiotics ineffective. Studies from different parts of India reported the different ESBL rates with 24.7\% ESBL rate in a study done by Khurana et al,20 31.8\% ESBL rate in a study by Purohit $M$ et al,21 34\% in a study conducted by Poovendran et al 22 and $41 \%$ of ESBL rate in a study done by Baby Padmini et al.23 These ESBL positivity rates in urinary isolates of $E$. coli correlate with figures of our study.

ESBL producing strains are creating significant therapeutic problems, since these pathogens are resistant to a wide range of $\beta$-lactams including third generation cephalosporins. In our study, it was found that resistance to cephalosporins in ESBL producing $E$. coli was coexisting with resistance to other antibiotics. The resistance to cotrimoxazole was between $59.8 \%-63.2 \%$, resistance to ciprofloxacin was between $67.2 \%-74.8 \%$, resistance to norfloxacin was between $47.2 \%-55.6 \%$ and gentamicin was between $39.2 \%$ - 44.5\% in all the ESBL producers in the years 2015 - 2017. The reason for this resistance is that ESBLs are encoded by plasmids, which also carry resistance genes for other antibiotics leading onto multidrug resistance. Multidrug resistance may be due to a number of factors like inappropriate self-medication, lack of prescribing regulations, substandard or falsified medicines and agricultural use of antibiotics. ${ }^{24}$
There was absolute resistance to beta-lactam-lactamase inhibitor combination drug amoxicillin-clavulanic acid. This is likely to be due to overuse of this drug. However, piperacillintazobactam was effective against ESBL producing $E$. coli with resistance rate ranging between $9.6 \%$ and $11.2 \%$ in the years 2015 - 2017. Carbapenems work by the inhibition of cell wall synthesis and are highly resistant to the beta-lactamase enzyme. In our study, meropenem was the most effective antibiotic against ESBL-producing $E$. coli. The resistance to Meropenem in ESBL producing E. coli was ranging between $9.4 \%-10.6 \%$ in our study. The lower resistance rate to carbapenems could be due to its usage pattern in our institution. It is most commonly used in critically ill patients where the administration of appropriate doses for the full duration is ensured. Surprisingly, resistance to nitrofurantoin in ESBL producing E. coli remains minimal in our study. It may be related to the fact that it has multiple mechanisms of action, hence may demand the organisms to develop more than a single mutation to concur resistance. 25

\section{CONCLUSION}

ESBL producing $E$. coli has become the most worrisome causative agent in UTI. One of the most worrisome aspects of ESBL positive $E$. coli concerns the high rate of resistance to non-beta lactam antibiotics, particularly quinolones, cotrimoxazole and aminoglycosides. The rising trend of ESBL production in $E$. coli is quite alarming as it limits the choices and outcome of antimicrobial treatment indicating the requirement of continuous monitoring systems and effective infection control measures.

\section{REFERENCES}

[1] Shakya P, Shrestha D, Maharjan E, et al. ESBL production among E. coli and Klebsiella spp. causing urinary tract infection: a hospital based study. The Open Microbiology Journal 2017;11:23-30.

[2] Dugal S, Purohit H. Antimicrobial susceptibility profile and detection of extended spectrum beta lactamase production by Gram negative uropathogens. International Journal of Pharmacy and Pharmaceutical Sciences 2013;5(4):434-8.

[3] Manisha MS, Bhat SK, Priya LR, et al. Prevalence and antibiotic susceptibility pattern of bacterial isolates from urinary tract infections in a tertiary care hospital in Tamilnadu. IOSR Journal of Dental and Medical Sciences (IOSR-JDMS) 2015;14(7):Ver. II. 59-65.

[4] Chauhan S, Mahawal BS, Ramola DC. Extended spectrum $\beta$-lactamases in urinary isolates of Escherichia coli - prevalence and susceptibility pattern at a tertiary care hospital. Int J Res Med Sci 2015;3(7):1622-6.

[5] Nicolle LE, AMMI Canada Guidelines Committee. Complicated urinary tract infection in adults. Can J Infect Dis Med Microbiol 2005;16(6):349-60.

[6] Picozzi SCM, Casellato S, Rossini M, et al. Extendedspectrum beta-lactamase-positive Escherichia coli causing complicated upper urinary tract infection: Urologist should act in time. Urology Annals 2014;6(2):107-12. 
[7] Qiao LD, Chen S, Yang Y, et al. Characteristics of urinary tract infection pathogens and their in vitro susceptibility to antimicrobial agents in China: data from a multicenter study. BMJ Open 2013;3(12):e004152.

[8] Ranjan KP, Ranjan N. Complicated urinary tract infection caused by extended spectrum $\beta$-lactamaseproducing Escherichia coli. Urology Annals 2014;6(2):112-3.

[9] Moura A, Nicolau A, Hooton T, et al. Antibiotherapy and pathogenesis of uncomplicated UTI: difficult relationships. Journal of Applied Microbiology 2009;106(6):1779-91.

[10] Arjunan M, Al-Salamah AA, Amuthan M. Prevalence and antibiotic susceptibility of uropathogens in patients from a rural environment, Tamilnadu. Am J Infect Dis 2010;6(2):29-33.

[11] Baral P, Neupane S, Marasini BP, et al. High prevalence of multidrug resistance in bacterial uropathogens from Kathmandu, Nepal. BMC Res Notes 2012;5:38.

[12] Bhatt C, Shrestha B, Khadka S, et al. Etiology of urinary tract infection and drug resistance cases of uropathogens. J Kathmandu Med Coll 2012;1(2(2):114-0.

[13] Singhal A, Sharma R, Jain M, et al. Hospital and community isolates of uropathogens and their antibiotic sensitivity pattern from a tertiary care hospital in north west India. Ann Med Health Sci Res 2014;4(1):51-6.

[14] Saha S, Nayak S, Bhattacharyya I, et al. Understanding the patterns of antibiotic susceptibility of bacteria causing urinary tract infection in West Bengal, India. Front Microbiol 2015;5:463.

[15] Chaudhari BK, Singh GK, Parajuli KP, et al. Incidence and susceptibility of uropathogens isolated among the patients at tertiary care hospital in Eastern Nepal. Journal of Nobel Medical College 2016;5(9):51-5.

[16] Sood S, Gupta R. Antibiotic resistance pattern of community acquired uropathogens at a tertiary care hospital in Jaipur, Rajasthan. Indian J Community Med 2012;37(1):39-44.
[17] Jeyasekharan DD, Manohar RN, Devaprasath R, et al. Long-term surveillance of Escherichia coli antibiotic resistance patterns in urinary tract infections in a southern India hospital. Astrocyte 2015;1(4):264-7.

[18] Kulkarni SR, Peerapur BV, Sailesh KS. Isolation and antibiotic susceptibility pattern of escherichia coli from urinary tract infections in a tertiary care hospital of north eastern Karnataka. Journal of Natural Science Biology, Medicine 2017;8(2):176-80.

[19] Saravanan R, Raveendaran V. Antimicrobial resistance pattern in a tertiary care hospital: an observational study. Journal of Basic and Clinical Pharmacy 2013;4(3):56-63.

[20] Khurana S, Taneja N, Sharma M. Extended spectrum beta lactamase mediated resistance in urinary tract isolates of family Enterobacteriaceae. Indian J Med Res 2002;116:145-9.

[21] Purohit M, Mutha A. Evaluation of Extended Spectrum Beta-Lactamase (ESBL) in urinary isolates of Escherichia coli and Klebsiella pneumoniae. J Evolution Med Dent Sci 2016;5(1):19-22.

[22] Ponnusamykonar P, Vidhya N, Murugan S. et al. Antimicrobial susceptibility pattern of ESBL and NonESBL Producing Uropathogenic Escherichia Coli (UPEC) and their correlation with biofilm formation. International Journal of Microbiological Research 2013;4(1):56-63.

[23] BabyPadmini S, Appalaraju B. Extended spectrum lactamases in urinary isolates of Escherichia coli and Klebsiella pneumoniae - prevalence and susceptibility pattern in a tertiary care hospital. Indian Journal of Medical Microbiology 2004;22(3):172-4.

[24] Singh N, Pattnaik D, Neogi DK, et al. Prevalence of ESBL in Escherichia coli isolates among ICU patients in a tertiary care hospital. Journal of Clinical and Diagnostic Research JCDR 2016;10(9):DC19-DC22.

[25] Shanmugam D, Esak SB, Narayanaswamy A. Molecular characterisation of nfsA gene in nitrofurantoin resistant uropathogens. Journal of Clinical and Diagnostic Research JCDR 2016;10(6):DC05-DC09. 